\title{
The Hofstadter Diagram Analyzed in Terms of the Band Structure Parameters for a Planar Metal
}

\author{
T. ROLiński AND S. OlszeWSKI \\ Institute of Physical Chemistry of the Polish Academy of Sciences \\ Kasprzaka 44/52, 01-224 Warsaw, Poland \\ (Received October 25, 2005; in final form January 27, 2006) \\ It is demonstrated that a wing of the Hofstadter diagram calculated for \\ the tightly-bound $s$-electrons in the simple cubic lattice can be reproduced \\ by the dispersion figure of the wave-vector square considered for the elec- \\ tron states of the same lattice, on the condition that the states having equal \\ energies are taken into account. The dispersion splitting increases system- \\ atically with the distance of the states from the Brillouin zone center. A \\ similar wing due to dispersion of the electron momentum is calculated also \\ for the $s$-electron states in the body-centered cubic lattice.
}

PACS numbers: 75.10.Lp, 75.70.--i

\section{Introduction}

In considering the electron motion in a planar metal submitted to the action of a constant magnetic field being normal to a crystallographic plane we meet the problem of a plot of the motion parameters versus the electron energy. If solely closed electron orbits are examined, a basic parameter entering the description of the electron motion is the ratio

$$
\alpha=\frac{\Phi}{\Phi_{0}} .
$$

The numerator $\Phi$ of (1) is the magnetic flux enclosed by the electron path, viz.

$$
\Phi=\int \boldsymbol{B} \cdot \mathrm{d} \boldsymbol{S},
$$

where $\boldsymbol{B}$ is the intensity of the magnetic field, $\mathrm{d} \boldsymbol{S}$ is the surface element; $\Phi_{0}$ in the denominator of (1) is the flux quantum

$$
\Phi_{0}=\frac{h}{e} .
$$


When the electron energy $E$ is plotted versus $\alpha$ we obtain, in general, an interval of $\alpha$ corresponding to each $E$ because of the dispersion relation between $\alpha$ and a given $E$. Graphically the plot leads to a figure called the Hofstadter butterfly because of its shape [1-6].

A quantum-mechanical calculation of $\alpha$ and its degeneracy is usually a rather complicated task. In fact, the problem seems to be solved and discussed only in the case of a planar $(0,0,1)$ lattice descending from the simple cubic (sc) crystal lattice for which the $s$-electrons in the tight-binding approximation are only considered. Beyond of the sc lattice some attempts to the solution of the Hofstadter problem for the tightly-bound $s$-electrons in a planar crystal structure are done also for the case of the body-centered cubic (bcc) and hexagonal lattice [3, 7-9]. Many effects, especially those connected with the Hall conductivity of the Bloch electrons in the planar structures and the de Haas-van Alphen oscillations, are examined with the reference to the Hofstadter diagram [3, 10].

The aim of the present paper is to replace an examination of the degeneracy problem of $\alpha$ performed on a quantum-mechanical basis by a semiclassical method. This approach can be developed beginning with the observation done recently by Analytis et al. [11] who considered a spinless electron particle moving along a circular ring lying in the $x y$ plane and having radius $r$. If a magnetic field $B_{z}$ perpendicular to the ring is applied, the ratio $\alpha$ provides us with the additional phase factor which is due to the magnetic field. This property holds for any angle $\varphi$ of the particle gyration and the phase factor becomes

$$
\exp (\mathrm{i} \alpha \varphi)=\exp \left(\mathrm{i} \frac{e}{\hbar} \frac{B_{z} r^{2} \varphi}{2}\right) .
$$

In consequence of (4), we have

$$
\alpha=\frac{e}{\hbar} \frac{B_{z} r^{2}}{2} .
$$

This is a very useful formula because $r^{2}$ is a readily accessible parameter in a semiclassical theory describing a crystal electron moving along a quasi-circular orbit in effect of the action of a constant magnetic field. It should be noted that the basic parameter concerning the motion in the field of $B_{z}$ is the electron gyration frequency which is proportional to $B_{z}$. On the other hand, the shape of the equations of motion of a crystal electron remains in fact independent of the size of $B_{z}$; see [12]. These equations provide us with a circular shape of the electron path along a ring which is given by a cross-section line of the Fermi surface with a plane $k_{z}=$ const already for rather small $B_{z}$. In consequence, the effective $B_{z}$ acting on an electron can be identified with $B_{z}$ entering the formula (4), and a momentary size of $r^{2}$ in the plane $(x, y)$ can be obtained by a projection of the wave vector square

$$
k^{2}=k_{x}^{2}+k_{y}^{2}
$$

calculated in the plane of the wave-vector components $k_{x}$ and $k_{y}$. The effect of 
projection is represented by the well-known formula [13]:

$$
k^{2}=\frac{e^{2} B_{z}^{2}}{\hbar^{2} c^{2}} r^{2} .
$$

For the electron energies being near the center of the band of states of a planar metal (see [11] and Sect. 2 below) it can be shown that

$$
k^{2} \longrightarrow 0
$$

and - simultaneously - $E$ becomes isotropic in the plane of $\left(k_{x}, k_{y}\right)$, viz.

$$
E=E\left(k^{2}\right) \text {. }
$$

On the other hand, for energies being far from the situations represented by (7) and (7a) a strong anisotropy of $E$ for different directions of the planar-wave vector

$$
\boldsymbol{k}=\boldsymbol{i} k_{x}+\boldsymbol{j} k_{y}
$$

is obtained. This provides us with an evident dispersion of the values of $k^{2}$, and therefore of $r^{2}$, corresponding to a given $E$. In effect - because of the formula (5) - a figure similar to a fragment of the Hofstadter butterfly demonstrated in Fig. 1 can be obtained with the aid of a plot of the electron energy versus parameter $\alpha$. The plot can be done not only for the energies of the tightly-bound $s$-electrons in the planar lattice descending from a sc case [1], but also for the energy spectra in other planar lattices, for example in a planar lattice descending from the bcc case. Our principal aim is to compare a fragment of the Hofstadter diagram calculated for the planar lattice descending from the sc case with a diagram coming from the dispersion calculations of the electron energy in the same lattice. For the sake of simplicity the lattice parameter entering the energy expressions is assumed equal to unity.

\section{Examination of the free-electron approximation}

First we demonstrate that the free-electron case provides us with zero dispersion of the planar electron momentum. The planar electron energy in this case is $(m=\hbar=1)$ :

$$
E^{\text {free }}=\frac{1}{2}\left(k_{x}^{2}+k_{y}^{2}\right)
$$

therefore

$$
k_{y}^{2}=\left(2 E^{\text {free }}-k_{x}^{2}\right)^{1 / 2} .
$$

An extremal value of $k_{y}$ versus $k_{x}$ is obtained at

$$
k_{x}=0,
$$

because the requirement

$$
\frac{\mathrm{d} k_{y}}{\mathrm{~d} k_{x}}=\frac{1}{2}\left(2 E^{\text {free }}-k_{x}^{2}\right)^{-1 / 2} 2 k_{x}=0
$$

is fulfilled solely at $k_{x}$ satisfying (11). Consequently, a substitution of (11) into

(10) gives only one number of $k_{y}>0$, viz.

$$
k_{y}=\left(2 E^{\text {free }}\right)^{1 / 2}
$$

for any $E^{\text {free }}$ and - from (11) and (13) - we obtain 
$k^{2}=k_{x}^{2}+k_{y}^{2}=2 E^{\text {free }}$.

Therefore, there is only one value of $k^{2}$ which satisfies (14) for the planar free-electron motion and, consequently, no dispersion of $k^{2}$ may exist in the free-electron case. In Sect. 3 and Sect. 4 we demonstrate that this situation does not apply for the tightly-bound $s$-electrons in planar crystal lattices for which a dispersion of $k^{2}$ corresponding to a given value of the planar electron energy can be easily calculated.

\section{Momentum dispersion for a planar crystalline structure descending from the simple cubic lattice}

The energy expression for the tightly-bound $s$-electrons in the three-dimensional (3D) sc lattice, viz. [14]:

$$
E_{(3 \mathrm{D})}^{\mathrm{sc}}=3-\cos k_{x}-\cos k_{y}-\cos k_{z},
$$

when considered in a plane of $k_{z}=$ const $=0$ is reduced to the planar electron energy equal to

$$
E^{\mathrm{sc}}=2-\cos k_{x}-\cos k_{y} .
$$

The constant 3 and a negative sign before $\cos k_{x}, \cos k_{y}$ and $\cos k_{z}$ entering in (15) are chosen in order to make valid at small $k_{x}, k_{y}$ and $k_{z}$ the following approximate relation for a 3D-lattice:

$$
E_{(3 \mathrm{D})}^{\mathrm{sc}} \approx E_{(3 \mathrm{D})}^{\mathrm{free}}=\frac{1}{2}\left(k_{x}^{2}+k_{y}^{2}+k_{z}^{2}\right) .
$$

A similar relation

$$
E^{\mathrm{sc}} \approx E^{\text {free }}
$$

holds also for a planar lattice $\left(k_{z}=0\right)$ at small $k_{x}$ and $k_{y}$. This free-electron character of energy is changed, however, for the electron states being far from the central point of the Brillouin zone.

With the hopping integral neglected, the Hofstadter energy $E^{\text {scHof }}$ for a planar sc lattice is

$$
E^{\text {scHof }}=2\left(\cos k_{x}+\cos k_{y}\right),
$$

so from (15a):

$$
E^{\mathrm{sc}}=2-\frac{1}{2} E^{\mathrm{scHof}} .
$$

In particular, for $k_{x}=k_{y}=\pi$ taken as an example we obtain

$$
E^{\mathrm{sc}}=2-\frac{1}{2} \times(-4)=4
$$

because $E^{\text {scHof }}=-4$; for the same length of the wave vector $|\boldsymbol{k}|$ but different signs of the vector components, for example $k_{x}=\pi, k_{y}=-\pi$, we have

$$
E^{\mathrm{sc}}=2-\frac{1}{2} \times 0=2
$$

because $E^{\text {scHof }}=0$. Our aim is to demonstrate that dispersion of the wave-vector length $|\boldsymbol{k}|$ obtained for the electron energies being far from the center of the Brillouin zone can provide us with a diagram much similar to the Hofstadter figure. To this purpose only the broadest right wing of one-fourth of the Hofstadter diagram presented in Fig. 1 which touches the energy line of $E^{\mathrm{sc}}=2$ is considered. 


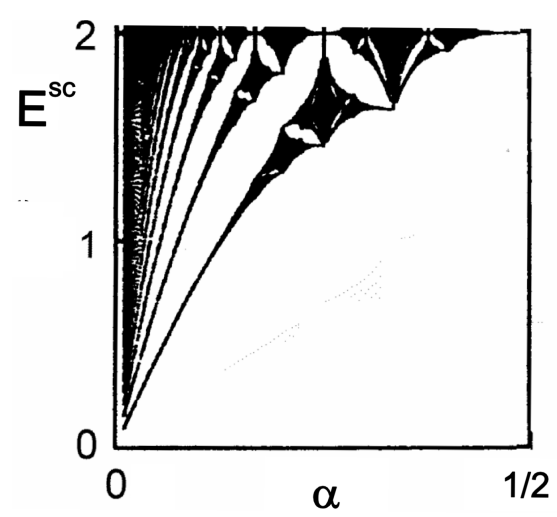

Fig. 1. A fragment (one-fourth) of the Hofstadter diagram obtained for the tightly-bound $s$-electrons in a planar structure of the sc lattice. The vertical variable is the electron energy, the horizontal variable is a dimensionless coefficient $\alpha=a^{2} e B / h c$ ( $a$ is the lattice parameter, $B$ is the strength of the magnetic field).

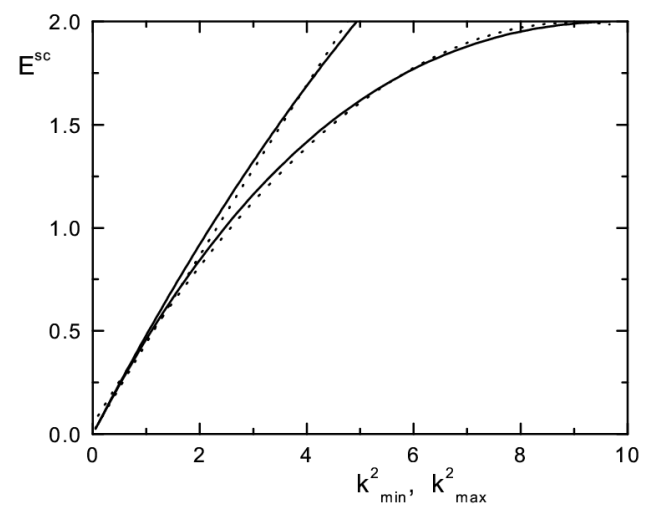

Fig. 2. A comparison of the rightmost wing of the Hofstadter diagram plotted in Fig. 1 with the dispersion of the wave-vector square $k^{2}$ : dashed lines are the limits of the Hofstadter wing of Fig. 1, the upper continuous line is a plot of $k_{\min }^{2}$ and the lower continuous line is a plot of $k_{\max }^{2}$ presented for different planar $s$-electron tight-binding energies $E=E^{\mathrm{sc}}$ in the sc lattice.

For the sc lattice the function

$$
k^{2}=k_{x}^{2}+k_{y}^{2}=k_{x}^{2}+\left[\cos ^{-1}\left(2-E^{\mathrm{sc}}-\cos k_{x}\right)\right]^{2}
$$

is examined for a constant $E^{\mathrm{sc}}$. This leads to the equation

$$
\frac{\mathrm{d}\left(k^{2}\right)}{\mathrm{d} k_{x}}=2 k_{x}+2 \cos ^{-1}\left(2-E^{\mathrm{sc}}-\cos k_{x}\right) \frac{\sin k_{x}}{\sqrt{1-\left(2-E^{\mathrm{sc}}-\cos k_{x}\right)^{2}}}=0 .
$$

One extremum (the maximum) of $k^{2}$ is at $k_{x}=0$. In this case

$$
k_{\max }^{2}=\left[\cos ^{-1}\left(1-E^{\mathrm{sc}}\right)\right]^{2} .
$$

The other extremum (the minimum) of $k^{2}$ can be obtained numerically by solving 
Eq. (18). A dispersion of $k^{2}$ defined by the difference

$$
\Delta\left(k^{2}\right)=\left(k_{\max }^{2}-k_{\min }^{2}\right)_{E^{\text {sc }}=\text { const }}
$$

is presented in the form of the limits $k_{\min }^{2}$ and $k_{\max }^{2}$ plotted as functions of $E=E^{\mathrm{sc}}$ in Fig. 2 together with the rightmost wing of the Hofstadter diagram. In order to make the arrangement of the diagram similar to that done by Hofstadter, the vertical line in the figure is chosen for energy and the horizontal line is taken for $k^{2}$.

\section{Momentum dispersion for a planar structure of the body-centered cubic lattice}

A similar dispersion of momentum characteristic of the sc lattice can be examined also in the bcc lattice case. The tightly-bound $s$-electrons considered in a three-dimensional bcc lattice [14], viz.

$$
E_{(3 \mathrm{D})}^{\mathrm{bcc}}=1-\cos k_{x} \cos k_{y} \cos k_{z}
$$

provide us in the plane $k_{z}=$ const $=0$ with the following expression for the planar electron energy:

$$
E^{\mathrm{bcc}}=1-\cos k_{x} \cos k_{y} .
$$

Here at $k_{x} \approx k_{y} \approx k_{z} \approx 0$ expression (22) tends again to the free-electron expression $E_{(3 \mathrm{D})}^{\text {free }}$ in $(16)$ and the planar expression (22a) tends to an expression similar to $(16 \mathrm{a})$, viz.

$$
E^{\mathrm{bcc}} \approx E^{\mathrm{free}}
$$

The function of energy calculated for the momentum square becomes

$$
k^{2}=k_{x}^{2}+k_{y}^{2}=k_{x}^{2}+\left[\cos ^{-1}\left(\frac{1-E^{\mathrm{bcc}}}{\cos k_{x}}\right)\right]^{2} .
$$

When examined for a constant $E^{\text {bcc }}$ in (22a), expression (24) gives the equation

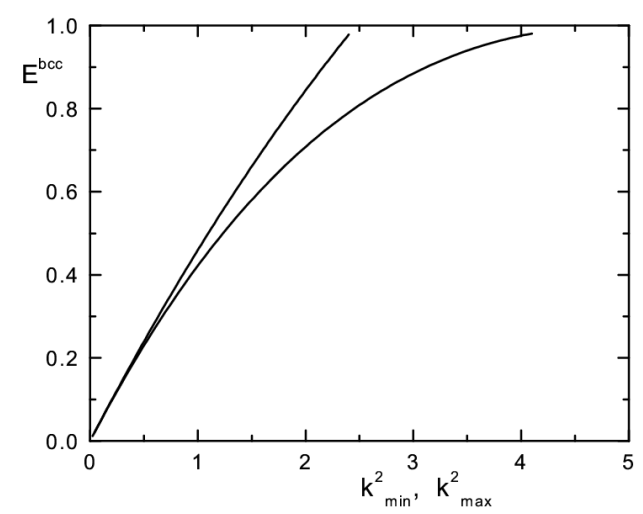

Fig. 3. A dispersion of the wave-vector square for the tightly-bound $s$-electron states in a plane of the bcc lattice for different planar energies $E^{\mathrm{bcc}}$ : upper curve $-k_{\min }^{2}$, lower curve $-k_{\max }^{2}$. 


$$
\frac{\mathrm{d}\left(k^{2}\right)}{\mathrm{d} k_{x}}=2 k_{x}+2 \cos ^{-1}\left(\frac{1-E^{\mathrm{bcc}}}{\cos k_{x}}\right)\left(-\frac{\sin k_{x}}{\cos ^{2} k_{x}}\right) \frac{1}{\sqrt{1-\left(\frac{1-E^{\mathrm{bcc}}}{\cos k_{x}}\right)^{2}}}=0 .
$$

One extremum (the maximum) of the function $k^{2}$ is attained at $k_{x}=0$ giving

$$
k_{\max }^{2}=\left[\cos ^{-1}\left(1-E^{\mathrm{bcc}}\right)\right]^{2},
$$

and the minimum of $k^{2}$ can be calculated by solving numerically Eq. (25). The dispersion of $k^{2}$ obtained in this way is plotted in Fig. 3.

\section{Summary}

We demonstrated for the tightly-bound $s$-electrons that dispersion of the wave-vector length calculated for the electron states having equal energies but located rather far from the central point of the Brillouin zone can provide us with a diagram much similar to the well-known Hofstadter diagram obtained for the sc lattice. A similar dispersion property can be shown to exist for the wave-vector lengths in the bcc lattice. In practical calculations only one wing of the Hofstadter diagram was considered, but other wings could be also reproduced in terms of the dispersion of the electron momentum on the condition that the energy scale is suitably changed.

\section{References}

[1] D.R. Hofstadter, Phys. Rev. B 14, 2239 (1976).

[2] D. Thouless, Phys. Rep. 110, 279 (1984).

[3] D. Springsguth, R. Ketzmerick, T. Geisel, Phys. Rev. B 56, 2036 (1997).

[4] G. Petschel, T. Geisel, Phys. Rev. Lett. 71, 239 (1993).

[5] T. Geisel, J. Wagenhuber, P. Niebauer, G. Obermair, Phys. Rev. Lett. 64, 1581 (1990).

[6] M.C. Chang, Q. Niu, Phys. Rev. B 53, 7010 (1996).

[7] H. Silberbauer, U. Rössler, Phys. Rev. B 50, 11911 (1994).

[8] O. Kühn, V. Fessatidis, H.L. Cui, P.E. Selbmaum, N.J.M. Horing, Phys. Rev. B 47, 13019 (1993).

[9] G. Gumbs, P. Fekete, Phys. Rev. B 56, 3787 (1997).

[10] O. Gat, J.E. Avron, Phys. Rev. Lett. 91, 186801 (2003).

[11] J.G. Analytis, S.J. Blundell, A. Ardavan, Am. J. Phys. 72, 613 (2004).

[12] S. Olszewski, T. Roliński, T. Kwiatkowski, Phys. Rev. B 59, 3740 (1999).

[13] J.C. Slater, Quantum Theory of Molecules and Solids, Vol. 3, McGraw-Hill, New York 1967.

[14] J.R. Reitz, in: Solid State Physics, Vol. 1, Eds. F. Seitz, D. Turnbull, Academic, New York 1955, p. 1. 